\title{
Real color captures attention and overrides spatial cues in grapheme-color synesthetes but not in controls
}

\author{
Tessa M. van Leeuwen ${ }^{\mathrm{a}, *, 1}$, Peter Hagoort ${ }^{\mathrm{a}, \mathrm{b}}$, Barbara F. Händel ${ }^{\mathrm{a}, \mathrm{c}, \mathrm{d}, * *, 1}$ \\ ${ }^{a}$ Radboud University Nijmegen, Donders Institute for Brain, Cognition and Behaviour, PO Box 9101, 6500 HB, Nijmegen, The Netherlands \\ ${ }^{\mathrm{b}}$ Max Planck Institute for Psycholinguistics, Wundtlaan 1, Nijmegen, The Netherlands \\ ${ }^{\mathrm{c}}$ Ernst Strüngmann Institute (ESI) for Neuroscience in Cooperation with Max Planck Society, Deutschordenstraße 46, 60528 Frankfurt am Main, Germany \\ ${ }^{\mathrm{d}}$ Maastricht University, Cognitive Neuroscience, Maastricht, The Netherlands
}

\section{A R T I C L E I N F O}

\section{Article history:}

Received 22 March 2013

Received in revised form

26 June 2013

Accepted 27 June 2013

Available online 4 July 2013

\section{Keywords:}

Synaesthesia

Attention

Colour

Alpha oscillations

Magnetoencephalography

\begin{abstract}
A B S T R A C T
Grapheme-color synesthetes perceive color when reading letters or digits. We investigated oscillatory brain signals of synesthetes vs. controls using magnetoencephalography. Brain oscillations specifically in the alpha band $(\sim 10 \mathrm{~Hz})$ have two interesting features: alpha has been linked to inhibitory processes and can act as a marker for attention. The possible role of reduced inhibition as an underlying cause of synesthesia, as well as the precise role of attention in synesthesia is widely discussed.

To assess alpha power effects due to synesthesia, synesthetes as well as matched controls viewed synesthesia-inducing graphemes, colored control graphemes, and non-colored control graphemes while brain activity was recorded. Subjects had to report a color change at the end of each trial which allowed us to assess the strength of synesthesia in each synesthete.

Since color (synesthetic or real) might allocate attention we also included an attentional cue in our paradigm which could direct covert attention. In controls the attentional cue always caused a lateralization of alpha power with a contralateral decrease and ipsilateral alpha increase over occipital sensors. In synesthetes, however, the influence of the cue was overruled by color: independent of the attentional cue, alpha power decreased contralateral to the color (synesthetic or real). This indicates that in synesthetes color guides attention. This was confirmed by reaction time effects due to color, i.e. faster RTs for the color side independent of the cue. Finally, the stronger the observed color dependent alpha lateralization, the stronger was the manifestation of synesthesia as measured by congruency effects of synesthetic colors on RTs.

Behavioral and imaging results indicate that color induces a location-specific, automatic shift of attention towards color in synesthetes but not in controls. We hypothesize that this mechanism can facilitate coupling of grapheme and color during the development of synesthesia.
\end{abstract}

(c) 2013 Elsevier Ltd. All rights reserved.

\section{Introduction}

In people with grapheme-color synesthesia, the percept of a specific letter or digit causes the additional experience of color, e.g. the letter J might elicit the color orange. The color percept is automatic, involuntary, and idiosyncratic (stable over time) (e.g. Baron-Cohen, Wyke, \& Binnie, 1987; Wollen \& Ruggiero, 1983).

\footnotetext{
* Corresponding author. Present address: Department of Neurophysiology, Max Planck Institute for Brain Research, Deutschordenstraße 46, 60528, Frankfurt am Main, Germany. Tel.: +496996769 240.

*** Corresponding author at: Ernst Strüngmann Institute (ESI) for Neuroscience in Cooperation with Max Planck Society, Deutschordenstraße 46, 60528 Frankfurt am Main, Germany.

E-mail addresses: tesvlee@gmail.com (T.M. van Leeuwen), barbara.haendel@esi-frankfurt.de (B.F. Händel).

${ }^{1}$ Equal contributors.
}

In grapheme-color synesthetes, brain activity to achromatic graphemes is enhanced in visual color area V4 (Hubbard, Arman, Ramachandran, \& Boynton, 2005; Sperling, Prvulovic, Linden, Singer, \& Stirn, 2006; van Leeuwen, Petersson, \& Hagoort, 2010) and in superior parietal cortex (Esterman, Verstynen, Ivry, \& Robertson, 2006; Weiss, Zilles, \& Fink, 2005). In superior parietal cortex, information from different modalities is integrated (Corbetta, Shulman, Miezin, \& Petersen, 1995; Robertson, 2003). Aberrant cross-activation of color area V4 to grapheme and/or parietal areas is proposed to underlie synesthetic color experience.

At least two possible mechanisms of cross-activation have been discussed. The cross-wiring hypothesis proposes that increased anatomical connections between the grapheme area and color area in ventral-occipital cortex might lead to an aberrant crossactivation between these brain areas (Brang, Hubbard, Coulson, Huang, \& Ramachandran, 2010; Ramachandran \& Hubbard, 2001a, 2001b; Rouw \& Scholte, 2007). Alternatively, the disinhibition 
model (Grossenbacher \& Lovelace, 2001) proposes functional changes in inhibition as a mechanism of synesthesia: after the combination of information in higher-order areas such as parietal lobe, feedback signals to lower sensory areas are disinhibited, leading to aberrant activation of color area V4. Evidence exists for both mechanisms. E.g. diffusion tensor imaging and voxel-based morphometry analyses have revealed anatomical differences in the synesthetes' brains, supporting the cross-wiring theory (Jäncke, Beeli, Eulig, \& Hanggi, 2009; Rouw \& Scholte, 2007; Rouw \& Scholte, 2010; Weiss \& Fink, 2009). However, synesthesia can be induced in non-synesthetes by training, posthypnotic suggestion or the application of drugs (Aghajanian \& Marek, 1999; Cohen Kadosh, Henik, Catena, Walsh, \& Fuentes, 2009; Hartman \& Hollister, 1963; Meier \& Rothen, 2009), suggesting that no abnormal anatomical connections are required to elicit synesthesia. Also, synesthetic experiences often resemble normal cross-modal associations (Bien, ten Oever, Goebel, \& Sack, 2012; Cohen Kadosh, Henik, \& Walsh, 2007; Sagiv, Simner, Collins, Butterworth, \& Ward, 2006; Ward, Huckstep, \& Tsakanikos, 2006), supporting the view that existing connections are functionally altered. In this paper, we address the question whether altered inhibitory processes may underlie synesthesia, using oscillatory activity in the brain as a marker for inhibition.

A second unsolved question is the role of attention in synesthetic percept. There is an ongoing discussion whether overt attention to the grapheme is necessary to elicit synesthesia or not. Behavioral studies have shown that synesthetic color can increase the efficiency of visual search tasks (Carriere, Eaton, Reynolds, Dixon, \& Smilek, 2009; Palmeri, Blake, Marois, Flanery, \& Whetsell, 2002; Ramachandran \& Hubbard, 2001a; Smilek, Dixon, \& Merikle, 2003; Ward, Jonas, Dienes, \& Seth, 2010), but this result has not been replicated in all cases (Edquist, Rich, Brinkman, \& Mattingley, 2006; Rothen \& Meier, 2009). Full attention does not seem to be necessary to elicit synesthesia (Rich \& Mattingley, 2003), however, true (pre-attentional) pop-out effects have not been reported. In the current study, we investigate the physiological underpinnings of attentional processes in synesthesia, aiming to see whether synesthesia requires full attention or not.

Brain oscillations specifically in the alpha band $(\sim 10 \mathrm{~Hz})$ as described by Berger (Berger, 1929) have two interesting features which might help to answer our questions: alpha power can act as a marker for attention and additionally has been linked to inhibitory processes. Alpha power is strongly modulated by attention showing a decrease in alpha power in areas that process the attended input and a power increase in areas that process distracting input (task-irrelevant areas). This has been observed in visual, auditory and somatosensory domains (Foxe, Simpson, \& Ahlfors, 1998; Fu et al., 2001; Haegens, Osipova, Oostenveld, \& Jensen, 2010; Jensen, Gelfand, Kounios, \& Lisman, 2002; Jokisch \& Jensen, 2007; Kelly, Lalor, Reilly, \& Foxe, 2006; Rihs, Michel, \& Thut, 2007; Van Dijk, Nieuwenhuis, \& Jensen, 2010; Worden, Foxe, Wang, \& Simpson, 2000; Yamagishi et al., 2003). Additionally, within one attentional state, alpha power is negatively correlated with perception. Low prestimulus alpha activity in visual areas is associated with good perceptual performance and fast reaction times to visual detection tasks, and vice versa (Ergenoglu et al., 2004; Hanslmayr et al., 2007; Thut, Nietzel, Brandt, \& PascualLeone, 2006; Van Dijk, Schoffelen, Oostenveld, \& Jensen, 2008; Zhang, Wang, Bressler, Chen, \& Ding, 2008). Such increases and decreases in alpha power seem actively controlled in order to follow the demands of the task at hand (Haegens, Handel, \& Jensen, 2011; Händel, Haarmeier, \& Jensen, 2011). The fact that a task is solved better if alpha oscillations are high in task irrelevant areas but low in task relevant areas leads to the belief that alpha reflects active inhibition of task-irrelevant regions and active disinhibition of task-relevant areas (Klimesch, Sauseng, \& Hanslmayr, 2007).

The disinhibition theory of synesthesia predicts that in synesthetes, alpha power would be decreased, reflecting reduced inhibition. Importantly, this decrease in alpha power should occur specifically for synesthesia-inducing stimuli. Besides testing this clear prediction, we further investigated the role of attention in synesthesia. Alpha lateralization can indicate the attentional allocation providing a tool to investigate attention during synesthetic color percept.

We measured the oscillatory brain activity of synesthetes and matched controls with magnetoencephalography (MEG). An attentional cueing task to assess attentional processes was combined with a color decision task (synesthetic Stroop task, Wollen \& Ruggiero, 1983) to assess behavioral effects of synesthesia and attention. Using alpha power as an indicator of inhibitory activity, we investigated whether synesthetes show any decreases in alpha power specifically for synesthesia-inducing stimuli. We further assessed whether there were any global or color-related differences in inhibitory processes for synesthetes compared to controls. Additionally, we investigated the attentional allocation during percept of non-colored, colored, and synesthesia-inducing graphemes.

\section{Methods}

\subsection{Participants}

Eleven grapheme-color synesthetes ( 9 female, mean age 27 years $(S D=3.8$ years), 2 left-handed) and 11 controls matched on age, handedness and educational level ( 8 female, mean age 25 years ( $S D=4.1$ years), 2 left-handed) participated in the study. The mean ages of the two groups did not differ $(t(10)=1.687$, n.s.) Developmental synesthesia was established by means of an extensive questionnaire on the synesthetes' experiences similar to the procedure in van Leeuwen, Petersson, \& Hagoort (2010). In the questionnaire synesthetes reported the color and intensity of their synesthesia for the 26 letters of the alphabet and digits 0-9. A surprise re-test on the 36 graphemes took place at least 2 months after the initial study (mean $5.5 \pm 2.5$ months) and yielded an average consistency score of $89 \%$ $(S D=8 \%)$, verifying genuine synesthesia (Baron-Cohen et al., 1987). Synesthetes differ in the way synesthetic color is perceived: 'projector' synesthetes experience color externally co-localized with a presented grapheme, whereas 'associators' report an internally evoked association of the color. Because individual differences can affect experimental outcomes (Dixon, Smilek, \& Merikle, 2004; Rouw \& Scholte, 2007; Rouw \& Scholte, 2010) nine specific questions on the location and shape of the synesthetic colors were used to characterize the synesthetes as projectors $(N=10)$ or associators $(N=1)$ (Rouw \& Scholte, 2007; van Leeuwen, den Ouden, \& Hagoort, 2011). Projector-associator scores ranged from -4 to 8 on a scale from -8 (associator) to 8 (projector), see van Leeuwen et al. (2011) for more details about this measure. Three out of ten projector synesthetes were classified as 'mental screen projectors' for whom the colors appear on a mental screen rather than in the exact location of the graphemes. Please note that these synesthetes have previously been classified as associators in some studies (e.g. Ward, Li, Salih, \& Sagiv, 2007) and as projectors in others (van Leeuwen et al., 2011, 2010).

Controls completed a pre-screening questionnaire to assess their medical history and handedness and were asked to associate a color with the 26 letters of the alphabet and digits 0-9. Unannounced re-testing of the color associations (after $2.5 \pm 2$ months on average) yielded an average consistency score of $34 \%$ $(S D=16 \%)$, which was significantly lower than the synesthetes' score; $t(20)=$ $10.125, p<.001$.

All participants had normal or corrected to normal vision, reported no color blindness and were easily able to discriminate the experimental colors. None reported a neurological or psychiatric disease. The study was performed in accordance with the Declaration of Helsinki and approved by the regional ethics committee for research on humans. Written informed consent was obtained from all participants prior to scanning.

\subsection{Experimental design}

The design of the study is summarized in Fig. 1. Trials consisted of two graphemes (letters and/or digits) presented left and right of a fixation cross. One of the graphemes was always displayed in dark gray and elicited no synesthesia for the synesthetes; these stimuli functioned as control stimuli and will be referred to as non-colored stimuli. The other grapheme could either be non-colored $(N$; i.e., presented in dark gray), physically colored but not eliciting synesthesia $(C)$, or noncolored eliciting synesthesia ( $S$; in synesthetes only). The stimulus conditions are referred to as 'non-color, 'color', and 'synesthesia' conditions, respectively. Please 


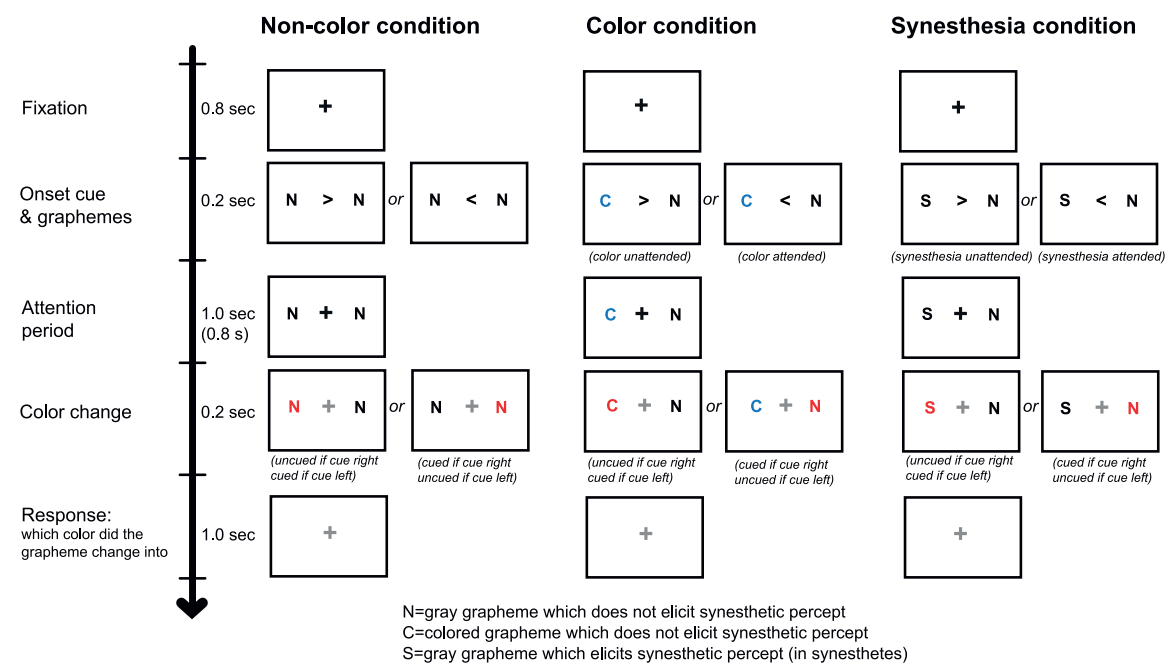

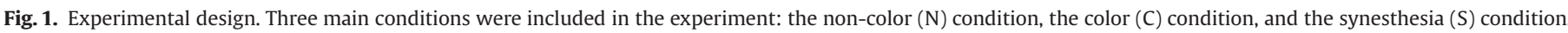

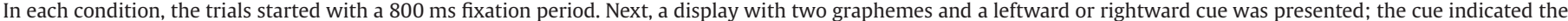

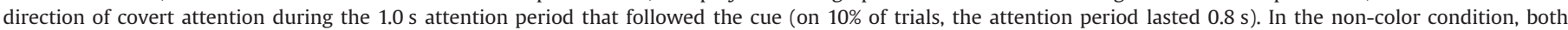

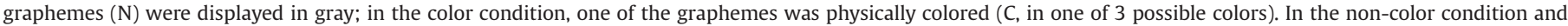

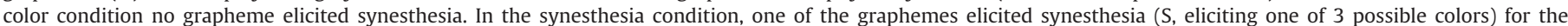

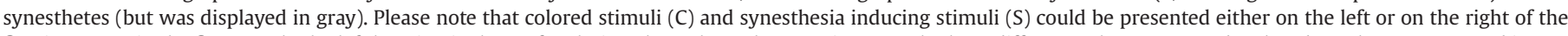

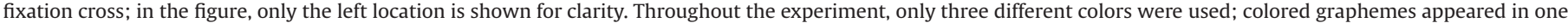

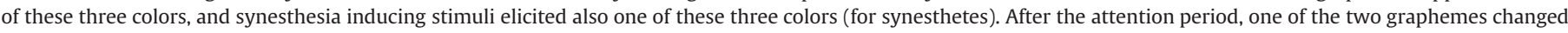

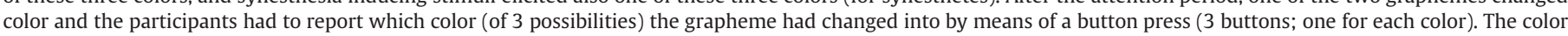

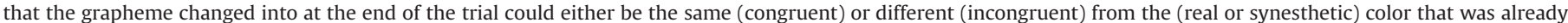

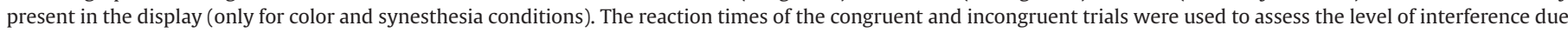

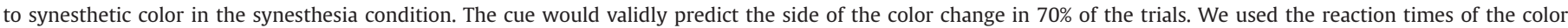
change task to assess the attentional allocation during the attention period.

note that for control participants, the 'synesthesia' condition was equivalent to the 'non-color' condition, as all graphemes were displayed in dark gray.

In Fig. 1, the time course of the trials is depicted. Each trial began with a fixation period of $800 \mathrm{~ms}$ where only the fixation cross was present. After $800 \mathrm{~ms}$, both graphemes and a directional cue (characters ' $<$ ' or ' $>$ ' indicating left or right, respectively) appeared. The cue was up for $200 \mathrm{~ms}$ and then was replaced by the fixation cross. The graphemes remained unchanged on the screen for either $1 \mathrm{~s}$ ( $90 \%$ of trials) or $800 \mathrm{~ms}$ (in $10 \%$ of trials). During this 'attention period', only the two graphemes and the fixation cross were present. Participants had to keep their gaze on the fixation cross and direct their covert attention to either the left or the right grapheme as previously cued. After the 'attention period' had finished the color of one of the graphemes changed into one of three possible colors (see Section 2.3 Stimuli and presentation settings) and participants had to report the physical color of the changed grapheme. Participants indicated the color by means of a button press (one button assigned to each of the three colors). The attentional cue was validly predicting the side at which the color change would happen in $70 \%$ of the trials and was invalid in $30 \%$ of trials; however, participants always had to respond. This allowed us to study the allocation of attention.

For synesthetes, the color decision task at the end of the trial could be used to assess behavioral effects of color interference due to synesthesia (Stroop, 1935) Specifically, in the synesthesia condition, the color that one of the graphemes changed into at the end of each trial could either be congruent (33\% of trials) or incongruent ( $67 \%$ of trials) with the synesthetic color that was present due to the synesthesia-inducing grapheme. This congruency manipulation has been widely used as a marker for synesthesia: reaction times to incongruent trials are usually longer than to congruent trials (e.g. Dixon et al., 2004; Wollen \& Ruggiero, 1983). In the real color condition, the color that one of the graphemes changed into could also be congruent $(33 \%)$ or incongruent $(67 \%)$ with the physical color of the grapheme that was displayed during the attention period. A congruent change in the color condition would mean that the color of the grapheme did not change at the end of the trial. Due to their different nature those trials were excluded from behavioral analysis. Because the congruency manipulation was included to specifically assess behavioral effects of synesthesia, we only analyze the congruency effects in the synesthesia condition.

Every color occurred equally often in every congruency condition and congruent trials were not overrepresented, avoiding possible behavioral strategy effects. Stimuli were randomized and presented in pseudorandom order with maximally 5 consecutive presentations of the same stimulus condition, left stimulus, right stimulus, cued side (attended/unattended), and color change side; maximally 3 consecutive presentations of invalid cues and early occurrences of the color change; and maximally 4 consecutive presentations of target color (and thus target button) and color congruency.
1296 trials were presented in 5 blocks of equal length which had short breaks in between. The actual MEG experiment took $\sim 70 \mathrm{~min}$. Prior to the MEG experiment participants performed a practice session that was monitored by the experimenter for speed and accuracy. Total experimental duration, including preparation time and an MR scan, was 2:30 h.

\subsection{Stimuli and presentation settings}

Because synesthesia is an idiosyncratic phenomenon, each synesthete required a unique stimulus set. Graphemes were chosen from the synesthesia questionnaire such that for each synesthete we could present 3 graphemes that elicited vivid synesthesia in 3 clearly distinct colors (for the synesthesia condition). Additionally 3 graphemes/symbols were chosen that elicited no synesthesia at all, also no achromatic synesthesia (used for the non-color and color conditions). Sometimes non-alphanumeric symbols were included (e.g. \#, \&) in case all letters of the alphabet and all digits elicited synesthesia. The control subjects received exactly the same stimulus list as the synesthete to whom they were matched.

Stimuli were presented against a light gray background (box of $21.5 \times 12 \mathrm{~cm}$, $456 \mathrm{~cd} / \mathrm{m}^{2}$ ), using Presentation software (version 13.0, Neurobehavioral Systems Inc., www.neurobs.com). Stimuli from the non-color and synesthesia conditions were presented in dark gray. The gray value of these stimuli was matched to the average luminance of the 3 colored stimuli (color condition) for each participant to avoid overall differences in luminance between stimulus conditions. Across all participants, the average luminance of the grapheme stimuli was $65 \mathrm{~cd} / \mathrm{m}^{2}$ $\left(S D=28 \mathrm{~cd} / \mathrm{m}^{2}\right)$.

All stimuli were $1.64^{\circ}$ of visual angle tall and were presented at positions $4.3^{\circ}$ to the left and right from the center of the screen $\left(35.5^{\circ} \times 27.9^{\circ}\right.$ of visual angle, placed at a viewing distance of $70 \mathrm{~cm}$ ). Stimulus presentation was controlled by a Dell Pentium IV Windows XP computer (display mode $800 \times 600$ pixels, $60 \mathrm{~Hz}$ ) and projected by a LCD projector (EIKI).

\subsection{MEG acquisition}

Neuromagnetic activity was recorded continuously at $600 \mathrm{~Hz}$ using a wholehead MEG system (CTF, Inc., Vancouver, Canada) containing 275 first-order axial magnetic gradiometers, situated at the Donders Institute for Brain, Cognition and Behaviour in Nijmegen, the Netherlands. Continuous data was low pass filtered at $150 \mathrm{~Hz}$, segmented into trials and further detrended. Line noise was attenuated using a $50 \mathrm{~Hz}$ notch filter. Horizontal and vertical EOG recordings were used for the rejection of artifacts that were introduced by eye movements or blinks, by means of 
a threshold detection ( $4 * z$-value). Muscle artifacts were removed by applying automatic artifact rejection in Fieldtrip.

\subsection{MEG data analysis}

All analyses were performed in MATLAB 7.5.0 (The MathWorks, Inc.) and the Fieldtrip software package (http://www.ru.nl/fcdonders/fieldtrip), a Matlab-based toolbox for the analysis of electrophysiological data. Only trials which fell between a threshold of \pm 7 times the mean variance were included in further analysis which resulted in on average 1158 trials ( $S D=152$ trials) for each participant. In order to optimize analysis over subjects on the sensor level, axial gradiometer information was converted into planar gradients (Bastiaansen \& Knosche, 2000; Van Dijk et al, 2008). The horizontal and vertical components of the planar gradients were estimated at each sensor location using the fields from the specific sensor and its neighboring sensors, and the power values for the horizontal and vertical components were summed after spectral analysis.

Time-frequency representations were calculated for the attention period using Fast Fourier Transforms (FFT). The analysis window had a length of $0.5 \mathrm{~s}$ which was shifted in steps of $0.1 \mathrm{~s}$, with a first centre point at $0.25 \mathrm{~s}$ after attention period onset (1.25 s after trial onset) and the last one at $0.25 \mathrm{~s}$ before the to be detected color change ( $1.75 \mathrm{~s}$ after trial onset). For low frequencies $(1-40 \mathrm{~Hz})$ a Hanning window was applied and frequency analyses started at $2 \mathrm{~Hz}$, increasing in steps of $2 \mathrm{~Hz}$. The high frequency power analysis $(40-120 \mathrm{~Hz})$ started at $40 \mathrm{~Hz}$ and increased in steps of $4 \mathrm{~Hz}$. It was estimated after applying 9 slepian tapers resulting in a frequency smoothing of $\pm 10 \mathrm{~Hz}$. Data was not baseline corrected and only correctly answered trials were included.

\subsubsection{Assessing effects of attention and general power differences}

Before testing our hypotheses by assessing differences in alpha power due to the synesthetic and real color conditions, we wanted to verify that alpha lateralization in the non-color condition followed the expected pattern. We further wanted to rule out any general alpha power differences between the groups. Therefore several independent tests were performed before continuing with the main analysis of the effects of real and synesthetic color.

First, to establish that the attentional cue had the expected lateralization effect on alpha power for both subject groups (decreased contralateral to attended side and increased ipsilaterally), we investigated the non-color condition calculating the normalized lateralization (i.e. cue to left side trials minus cue to right side trials divided by the sum of those trials) for left vs. right sensors. For each group, 5 occipito-parietal sensors with the highest absolute power over left and right hemisphere were selected and alpha power for left vs. right sensors was compared by means of a 2-sided paired $t$-test. Using normalized lateralization measures and a group based sensor selection is a common approach to show alpha lateralization (Händel et al., 2011; Thut et al., 2006). Please note that a group based sensor selection was only used for this specific analysis. For all further analyses, alpha power over all occipital sensors was analyzed rather than over a group based sensor selection in order to include the same sensors in all conditions and both groups (please note that including all sensors is a more conservative method).

Next, to exclude possible global differences in alpha power between synesthetes and controls, the two groups were directly compared in baseline alpha activity. For this analysis, mean power values measured during the baseline period ( 0.25 and $0.75 \mathrm{~s}$ after trial onset, fixation cross) in all occipital sensors were compared between groups by means of a 2-sided two-sample $t$-test.

Since there is also a possibility that synesthetes and control subjects would show differences in alpha power only during synesthetic percept we compared alpha activity during the attention period (1.25 s to $1.65 \mathrm{~s}$ after trial onset) in occipital sensors contralateral to the cued synesthetic stimulus between groups using a 2-sided two-sample $t$-test

\subsubsection{Alpha power lateralization due to real and synesthetic color}

After assessing alpha lateralization introduced by the attentional cue only, and after assessing possible general group differences in alpha power, we set out to analyze the synesthetic color and real color condition in more detail. We investigated whether the lateralization of alpha power differed between the experimental conditions and between groups. We contrasted trials from the color and synesthesia conditions with trials from the non-colored condition in the same attentional state (i.e. either attended or unattended). Thus, attended and unattended stimuli were analyzed separately, as well as trials in which the colored/ synesthetic stimulus was presented on the left vs. on the right. Power values during the attention period (1.25 s to $1.65 \mathrm{~s}$ after trial onset, i.e. $400 \mathrm{~ms}$ ) from occipital sensors that were located over the right hemisphere were averaged and contrasted with averages from sensors over left occipital cortex. The approach offered a measure of lateralization strength but was not normalized in order to preserve the individual differences in absolute power. This lateralization value served as the dependent variable in a 4-way factorial repeated measured ANOVA with the within-subject factors color type (synesthetic/real), attention (attended/unattended), and color side (left presentation/right presentation of color) and the between-subject factor group (synesthetes/controls).

\subsubsection{Correlation analysis}

We further investigated whether the strength of behavioral color interference that was induced by synesthesia correlated with the lateralization strength of alpha power over occipital sensors. This analysis was performed only for the synesthesia condition. For the lateralization strength of alpha power we used the difference in alpha power between left and right occipital sensors for the trials in which the synesthesiainducing stimuli were present opposite to the attended hemifield. The side of attention therefore does not coincide with the side of synesthetic color. The exclusion of one outlier was based on a threshold of 2.5 STD away from the mean.

\section{Results}

\subsection{Behavioral results}

Reaction times (RTs) from all 22 participants ( 11 synesthetes, 11 controls) were entered into analyses of variance (ANOVA). Misses amounted to $2.4 \%(S D=4.8 \%)$ of trials for synesthetes, and $3.2 \%$ $(S D=3.1 \%$ ) for controls (no group difference, $t(20)=-0.476$, n.s.). Outliers were removed prior to analysis by removing all RTs that were faster than $250 \mathrm{~ms}$ or slower than $2000 \mathrm{~ms}$ (synesthetes, $1.9 \%$; controls, $0.4 \%$, no group difference, $t(20)=0.906$, n.s.). Only correct responses were included in the analyses.

Overall accuracy was $89.6 \%(S D=10.2 \%)$ for the synesthetes and $90.9 \%(S D=9.5 \%)$ for the control participants and did not differ between groups $(t(20)=-0.304$, n.s.). Overall RTs did not differ between the groups: $F(1,20)=0.012$, n.s. Within the separate groups, there were no overall RT differences between the three stimulus conditions (synesthesia, non-color, color).

\subsubsection{Attention manipulation-Effect of cue validity}

The first behavioral analysis concerns the attention manipulation in our paradigm. To assess whether participants were correctly following the attentional cue, we analyzed reaction times to validly and invalidly cued trials for the three main experimental conditions (non-color, synesthetic color, real color). If attending correctly to the cued side, participants' reaction times should be slower on invalidly cued compared to validly cued trials. We performed a repeated measures ANOVA with the within-subject factors color type (non-color, synesthetic color, real color) and cue validity (valid and invalid), and the between-subject factor group (synesthetes and controls). For the synesthetic color and real color conditions, we collapsed the reaction times across the factors color congruency and side of attention. We found a main effect of cue validity $(F(1,20)=14.652, p<0.001)$. There was no main effect of color type $(F(2,40)=0.684$, n.s.) and there were no interactions (color type $\times$ group, $F(2,40)=0.563$, n.s.; cue validity $\times$ group, $F(1,20)=1.474$, n.s.; color type $\times$ cue validity, $F(2,40)=$ 0.716 , n.s.; color type $\times$ cue validity $\times$ group, $F(2,40)=0.125$, n.s.). Fig. 2A shows the reaction times for validly and invalidly cued trials in the non-color condition for both groups (synesthetes: valid cue $698 \pm 93 \mathrm{~ms}$, invalid cue $718 \pm 97 \mathrm{~ms}$; controls: valid cue $694 \pm 126 \mathrm{~ms}$, invalid cue $735 \pm 138 \mathrm{~ms}$ ). A post hoc paired sample $t$-tests confirmed a significant effect for both groups (synesthetes: $t(10)=-3.429, p<0.01$, controls $t(10)=-3.264, p<0.01)$, establishing the expected effect of attention in this control condition. In the synesthetic color condition the reaction times were as follows: synesthetes: valid cue $704 \pm 103 \mathrm{~ms}$, invalid cue $725 \pm 111 \mathrm{~ms}$ (post hoc $t$-test $t(10)=-2.433, p<0.05)$; controls: valid cue $699 \pm 129 \mathrm{~ms}$, invalid cue $737 \pm 147 \mathrm{~ms}$ (post hoc $t$-test $t(10)=-2.605, p<0.05$ ). In the color condition, the reaction times were: synesthetes: valid cue $716 \pm 92 \mathrm{~ms}$, invalid cue $731 \pm 105 \mathrm{~ms}$ (post hoc $t$-test $t(10)=-1.327$, n.s.); controls: valid cue $701 \pm 139 \mathrm{~ms}$, invalid cue $730 \pm 163 \mathrm{~ms}$ (post hoc $t$-test $t(10)=-2.034, p=0.069)$.

Informed by the later on described results on alpha-activity, that suggested altered attentional responses to color in synesthetes, we further inspected the reaction times for unattended color stimuli separately (Fig. 2B). A two-sided informed t-test showed that the 

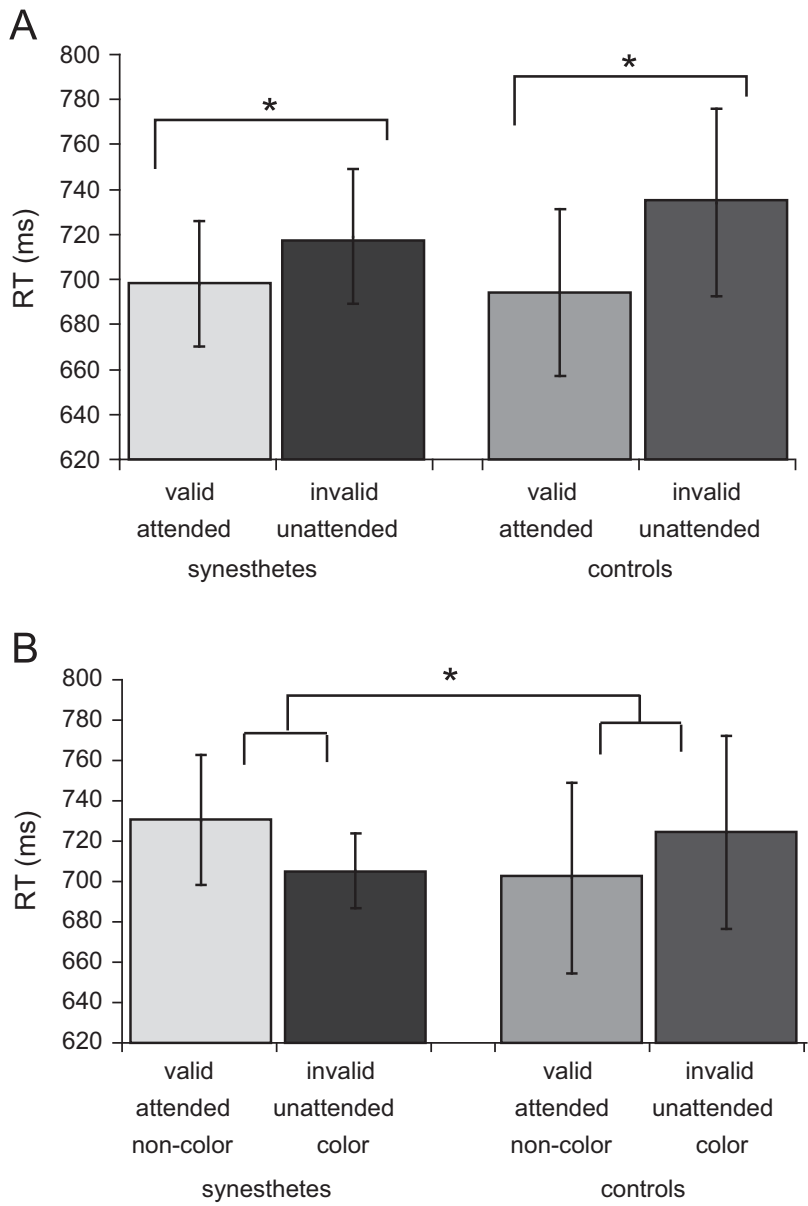

Fig. 2. Reaction time effects of the attentional manipulation. (A) Effects of attentional cue in the non-color condition for synesthetes (left) and controls (right). There is a significant increase in reaction times to color changes on the unattended (invalidly cued) versus the attended (validly cued) side. (B) Effects of attentional cue in the color condition. The results are displayed only for those trials in which the color was present on the unattended, non-cued side. The expected result is therefore that the reaction times to color changes that happen on the side of the colored stimuli (not cued) will be slower than the RTs to color changes happening on the side of the cued non-colored stimuli. Reaction times for both non-colored and colored stimuli are shown. For the controls, the expected pattern emerges: reaction times are slower on the unattended, non-cued but colored side (right panel). Synesthetes, however, are faster for the non-cued but colored stimuli (left panel): synesthetes orient their attention based on the position of the colored stimulus and not the cue. The RT difference between validly and invalidly cued trials significantly differs between synesthetes and controls (one-sided $t(10)=$ $1.745, p<0.05$ ). Error bars denote the standard error of the mean (SEM). * indicates a significant $(p<0.05)$ difference.

influence of the cue in the color condition was not the same for controls and synesthetes $(t(20)=2.194, p<0.05)$. In synesthetes, RTs for the invalidly cued color stimuli were faster than those for validly cued non-colored stimuli $(26 \pm 53 \mathrm{~ms}$ faster). This means that synesthetes were faster to react to color changes when they occurred on the side where color was present during the trial, even when this side was not cued. Controls were slower for the invalidly cued trials even if the stimulus was colored ( $21 \pm 49$ ms slower). Fig. 2B clearly demonstrates the opposite RT patterns of synesthetes and controls. This result also explains the absence of a significant effect of cue validity for the color condition in the synesthetes.

\subsubsection{Synesthetic color congruency manipulation in the color} decision task

In a second analysis which was orthogonal to the attentional manipulation, we assessed the behavioral effects of synesthesia by

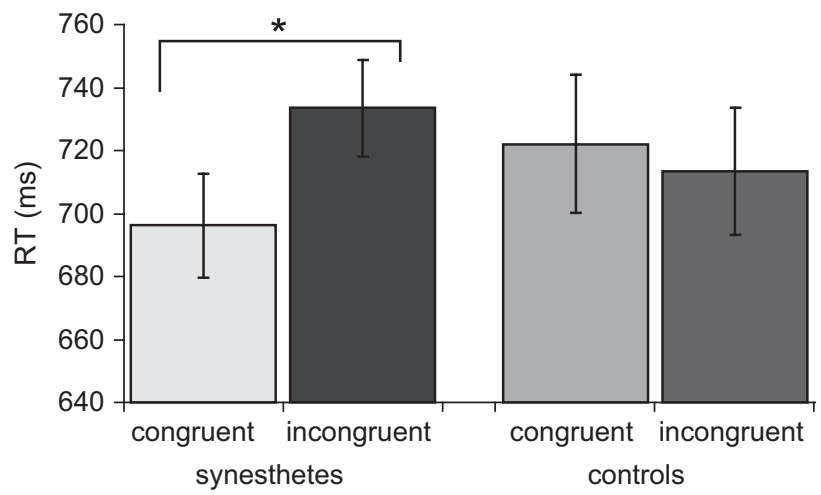

Fig. 3. Reaction time effect of synesthetic color congruency. Effects of synesthetic color congruency in color decision task in the synesthesia condition. We expected synesthetes to be slower to indicate the correct color in case this target color did not match with the synesthetic color that was induced during the trial (incongruent color). Synesthetes (left diagram) indeed showed significant slower reaction times if the presented color was incongruent with the induced synesthetic color Controls (right) showed no effect of color in the synesthesia condition. Error bars denote the standard error of the mean. * indicates a significant $(p<0.01)$ difference.

means of the congruency manipulation in our behavioral task. Because we aimed to identify the behavioral effects of synesthesia, we only assessed the congruency effects in the synesthesia condition and not in the color condition (also because congruent changes in the color condition were difficult to detect since effectively there was no change in color-see Section 2.2). For the synesthesia condition, we expected only synesthetes (and not controls) to be slower on incongruently colored trials, due to color interference effects caused by their synesthetic color percept. We performed a repeated measures ANOVA for the synesthesia condition with the within-subject factors congruency (congruent/ incongruent) and attention (attended/unattended stimuli), and the between-subject factor group (synesthetes/controls). An interaction of congruency $\times$ group was found $(F(1,42)=6.739, p<0.05)$, see Fig. 3. Exploring the interaction further, we found that synesthetes were $37 \mathrm{~ms}$ slower on incongruent vs. congruent trials $(F(1,21)=5.550, \quad p<0.05)$, while controls showed no effects $(F(1,21)=1.199$, n.s. $)$. There were no significant interactions or main effects involving attention (all $F<1.7$ ). This absence of an influence of attention implies that congruency effects were similar, regardless of whether synesthesia inducing graphemes were fully attended or not. Post hoc paired-sample $t$-tests confirmed a significant congruency effect for synesthetes for the trials in which the synesthesia-inducing stimulus was not attended $(t(21)=$ $-2.373, p<0.05)$ as well as a trend in the same direction for attended trials $(t(21)=-1.899, p=0.071)$.

\subsection{MEG results}

We inspected the time frequency representations of all conditions to assess oscillatory power differences. High gamma activity was visible for both groups, but no significant lateralization effects were found for the gamma band, possibly due to the lower signalto-noise ratio in the higher frequencies. As can be seen in Fig. 4A, for both synesthetes and controls the strongest modulation in oscillatory power due to color input was present in the alpha band. Therefore, all analyses were conducted on power values between 8 and $12 \mathrm{~Hz}$.

\subsubsection{Establishing alpha lateralization due to attention}

We first established that synesthetes and controls showed the expected alpha power lateralization in the non-color condition 
A

SYNESTHETES

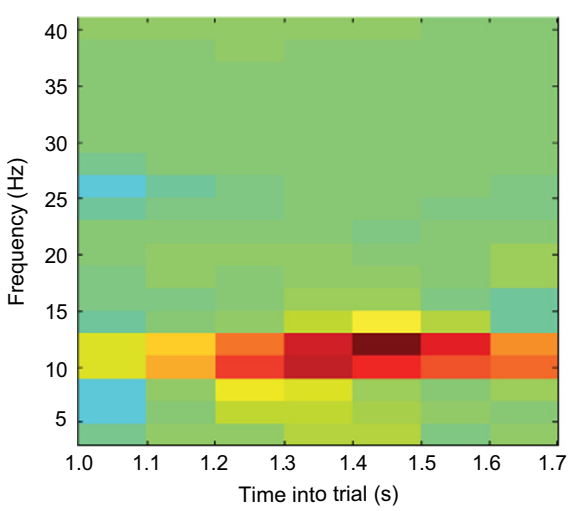

CONTROLS

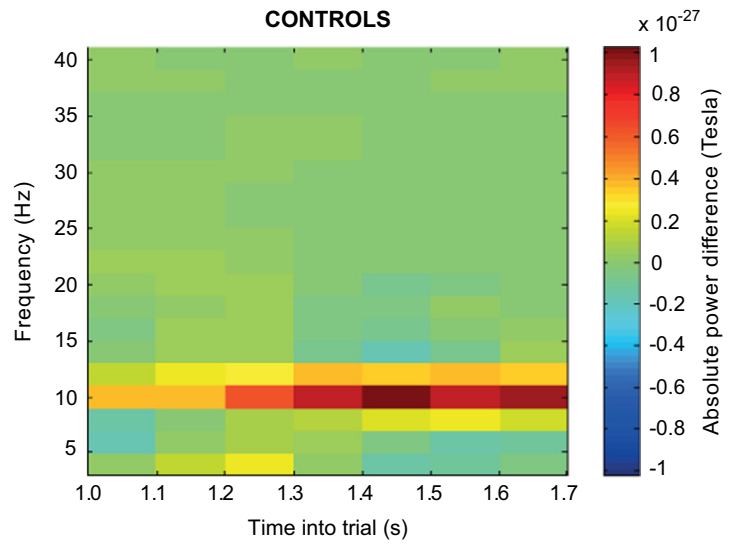

B
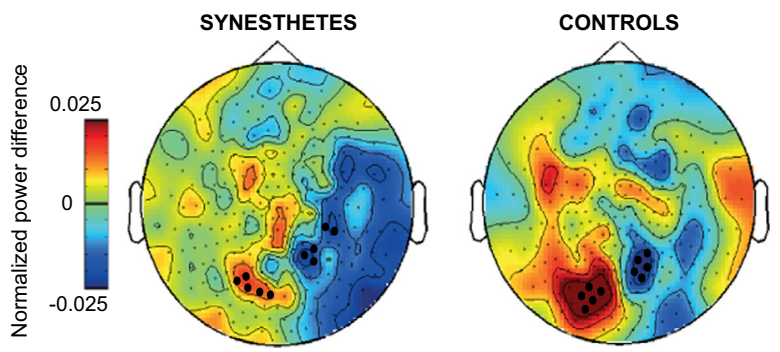

C
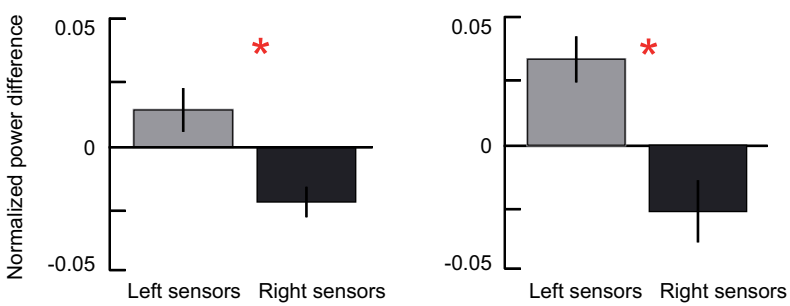

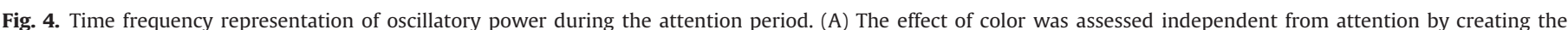

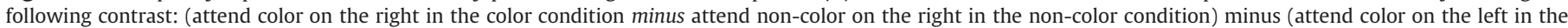

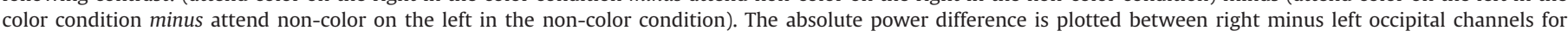

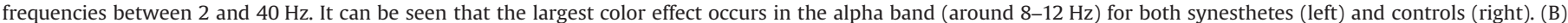

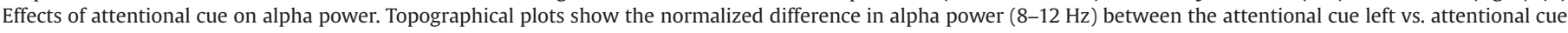

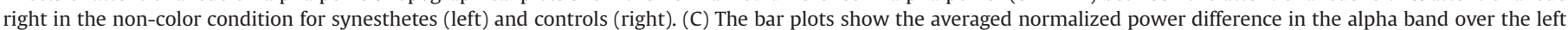

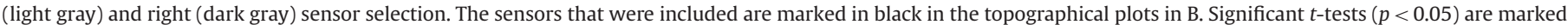
with an asterisk.

due to the allocation of attention as directed by the attentional cue. Using a normalized lateralization measure and selecting the 5 occipito-parietal sensors with the highest absolute power over left and right hemisphere for each group (sensors are marked by asterisks in Fig. 4B), we confirmed the expected effect of the attentional cue on alpha power for the non-color condition in both groups (synesthetes $t(10)=2.61, p=0.026$; controls $t(10)=4.28$, $p=0.0016)$. This result is displayed in Fig. $4 \mathrm{~B}$ and C. The alpha lateralization patterns are in line with the behaviorally established attentional cueing effects (Fig. 2A).

\subsubsection{General alpha power effects between groups}

We performed several independent tests to assess possible general alpha power differences between the two groups. We wanted to investigate the possibility that alpha power would already differ during baseline between synesthetes and control subjects. We therefore compared alpha power in all occipital sensors during the baseline period ( 0.25 and $0.75 \mathrm{~s}$ after trial onset, fixation cross). No significant group differences were found $(t(20)=-0.91, p=0.385$; mean control $=7.96^{-27}$, mean synesthetes $=5.6^{-27}$ ).

Next, we investigated whether synesthetes and control subjects would differ in their alpha power due to synesthetic percept. Synesthetic stimuli did not elicit different alpha power during their presence ( $1.25 \mathrm{~s}$ to $1.65 \mathrm{~s}$ after trial onset; attention period) in occipital sensors contralateral to the cued synesthetic stimulus (two-sample $t$-test, sensors left/stimulus right: $t(20)=0.185$, $p=0.86$; mean controls $=-0.015$, mean synaesthetes $=-0.0111$; sensors right/ stimulus left: $t(20)=-0.631, p=0.54$, mean controls $=-0.0165$, mean synaesthetes $=-0.0264$ ).

\subsubsection{Alpha power lateralization due to real and synesthetic color} depending on the attentional cue

A 4-way repeated measures ANOVA with the within-subject factors color type (synesthetic/real), attention (attended/unattended), and color side (left presentation/right presentation of color) and the between-subjects factor group (synesthetes/controls) was performed. Averaged power values during the attention 
period from occipital sensors located over the right hemisphere were contrasted with averages from sensors over left occipital cortex and served as the dependent variable. For the alpha power during the attention period, a significant three-way interaction of group $\times$ attention $\times$ color side was observed $(F(1,10)=8.282$, $p=0.016)$ and a significant three-way interaction of color type$\times$ attention $\times$ color side $(F(1,10)=602.352, p<0.001)$. Furthermore, we found a 2-way interaction of attention and color side $(F(1,10)=23.467, p<0.001)$. There was no 4-way interaction $(F(1,10)=1.951$, n.s.), there were no further 3 -way interactions (all $F<0.5$ ), no further 2-way interactions (all $F<2.9$ ), and no main effects (all $F<1$ ).

We followed up on the significant 3-way interaction of group$x$ attention $\times$ color side by looking at the effects for each group separately, performing two 2-way repeated measures ANOVAs with the factors attention (attended/unattended) and color side (left/right), collapsing across color type (synesthetic/real). We found a significant interaction of attention $\times$ color side for the synesthetes $(F(1,10)=10.34, p<0.01)$ but not for the controls $(F(1,10)=4.04, p=0.072$ ), and no main effects (all $F<2$ ). This finding indicates that only for the synesthetes the side where the colored stimulus is would influence the effects exerted by the attentional state (attended/unattended). Testing the simple effects from the 2-way ANOVA (factors: attention $\times$ color side) for the synesthetes, we can show that when stimuli are attended, significant alpha lateralization occurs for colored stimuli both on the left $(t(1,21)=3.11, p<0.01)$ and right side of the screen $(t(1,21)=-$ $2.58, p<0.05)$. For unattended stimuli, there is a significant lateralization of alpha only when the colored stimulus is on the left $(t(1,21)=2.57, p<0.05)$.

Our findings are summarized in Fig. 5 and discussed in detail below. To be able to inspect the nature of the lateralization effects, Fig. 5 shows the influence of color separately for all factors within each group. We plot alpha power induced by a (synesthetic or real) colored grapheme of which the alpha power induced by a noncolored grapheme is subtracted. We did this separately for the attended and the unattended state, as well as for the presentation in the left and right visual hemifield. We would like to emphasize that no direct interaction between group and color type (synesthetic vs real color) was found in the 4-way ANOVA. Due to the absence of this interaction we cannot say anything conclusive about differential effects in the real color and synesthetic color condition in the two groups-the effects are similar for both experimental conditions. However, since the phenomenon of synaesthesia is not there for the control participants, and the factor color type was a part of the other significant 3-way interaction in the 4-way ANOVA, we decided to separate the results for the synesthesia and the color condition in Fig. 5 nonetheless, for illustration purposes. Fig. 5 is described in Section 3.2.3.1 (color condition) and Section 3.2.3.2 (synesthesia condition) and the results are discussed in the light of the attention $\times$ color side interaction that we found for the synesthetes.

3.2.3.1. Alpha power effects in the color condition. Inspecting the nature of the alpha lateralization effects, we first looked into the color condition. In the top row (A) in Fig. 5 the results are displayed for the contrast between the attended color grapheme and the attended non-color grapheme displayed on the left side or the right side of the fixation cross. Synesthetes (left-hand side of Fig. 5A) show a clearly lateralized pattern over occipital sensors with a negative value contralateral to the attended color stimulus and a positive value ipsilateral. This leads to a strong alpha lateralization in occipital sensors due to colored stimuli (as highlighted by the bar plot) on the left and on the right. This finding indicates that for synesthetes a stronger alpha lateralization occurs when color is attended compared to if a non-color stimulus is attended. Controls (right-hand side of Fig. 5A) show a global decrease in alpha power when color is present, irrespective of the position of the colored stimulus.

When we subtracted the unattended non-colored stimulus from the unattended color grapheme (middle row (B) in Fig. 5), synesthetes again show a lateralization of alpha power. However, now there is a decrease in alpha power ipsilateral to the attended side but contralateral to the colored stimulus and an increase ipsilateral to the colored stimulus. Therefore, alpha power in synesthetes is affected by the colored stimulus in such a way that when color is presented opposite to the cue the effect of the cue is either greatly reduced or even reversed. This finding is in line with our behavioral results which showed that in synesthetes, reaction times to invalidly cued but colored stimuli were faster than those for validly cued, non-colored stimuli (in the color condition). For controls, the behavioral pattern followed the cue and the presence of color again led to a general reduction in alpha power (topographical plots on right-hand side in Fig. 5B). The bar plots confirm this general decrease in alpha power for both contralateral and ipsilateral sensors. When attention is directed to the right, alpha power is decreased more on the right side than on the left side, but both hemispheres still show a decrease in alpha power. This pattern is clearly distinct from the lateralization pattern shown by the synesthetes since an alpha increase due to color was clearly absent in the control group. This increase of alpha power ipsilateral to the colored stimulus in the synesthetes even when the colored stimulus was not cued, is a qualitative difference between synesthetes and controls showing the greater influence of the color side on attention for synesthetes.

3.2.3.2. Alpha power effects in the synesthesia condition. We inspected the alpha lateralization effects in the synesthesia condition separately. We investigated effects of synesthetic color percept on alpha power by contrasting the synesthesia condition with the non-color condition, again separately for each attended side. Fig. 5C (left side of bottom row in Fig. 5) illustrates that for synesthetes, the alpha lateralization patterns for attended synesthesia-inducing graphemes resemble those for physically colored graphemes. We found a decrease in alpha power contralateral to the attended synesthesia-inducing stimulus (compared to an attended non-color stimulus), and an ipsilateral increase. The effects appear less pronounced than in the real color condition. Controls also show some lateralization for synesthesia inducing stimuli, but as indicated by the bar plots, there is no clear ipsilateral increase for the synesthesia inducing stimuli as is the case for the synesthetes.

We also analyzed alpha power for synesthesia-inducing stimuli on the unattended side, and compared it to alpha power for noncolored stimuli on the unattended side. Again, synesthetes reduced alpha power contralateral to the synesthesia-inducing stimulus (plots not shown), indicating that the synesthesia inducing stimulus attracted attention. However, the behavioral data show no reversal of the reaction time patterns for validly vs invalidly cued trials, suggesting this effect was not strong enough to overrule the directional attention cue as was the case in the color condition.

\subsubsection{Correlation between alpha lateralization and strength of synesthesia}

To see whether the prevalent alpha lateralization due to color was related to the condition of synesthesia we investigated whether the strength of synesthesia correlated with the strength of the increased alpha lateralization due to unattended synesthesia-inducing stimuli. Fig. 6 shows a significant negative 


\section{SYNESTHETES}

\section{CONTROLS}

A Color $(\mathrm{C})$ attended minus non-color $(\mathrm{N})$ attended

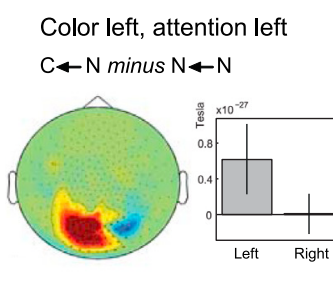

Color right, attention right

$\mathrm{N} \rightarrow \mathrm{C}$ minus $\mathrm{N} \rightarrow \mathrm{N}$
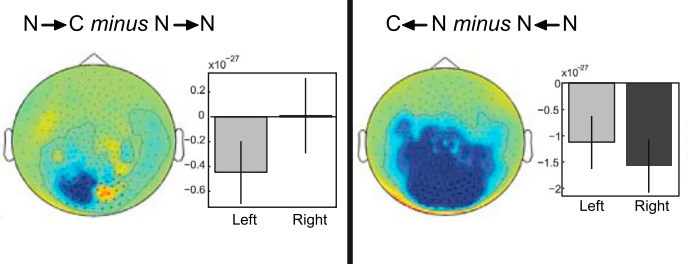

Color left, attention left

B Color (C) unattended minus non-color (N) unattended

\section{Color right, attention left}

$\mathrm{N} \leftarrow \mathrm{C}$ minus $\mathrm{N} \leftarrow \mathrm{N}$

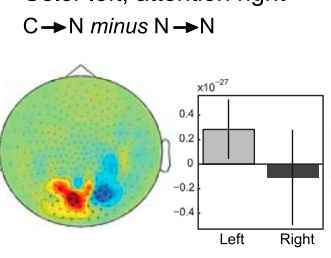

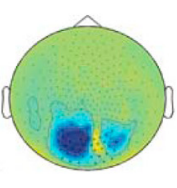

Color left, attention right $\mathrm{C} \rightarrow \mathrm{N}$ minus $\mathrm{N} \rightarrow \mathrm{N}$$$
4
$$

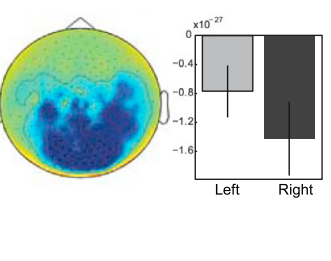

Color right, attention right

$\mathrm{N} \rightarrow \mathrm{C}$ minus $\mathrm{N} \rightarrow \mathrm{N}$

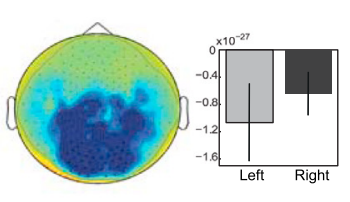

Cynesthesia inducing stimulus (S) attended minus non-color $(\mathrm{N})$ attended

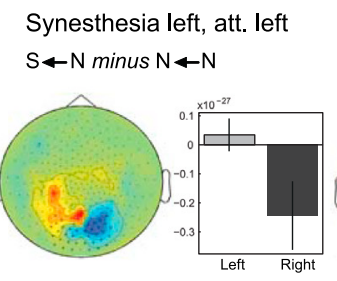

Synesthesia right, att. right $\mathrm{N} \rightarrow \mathrm{S}$ minus $\mathrm{N} \rightarrow \mathrm{N}$

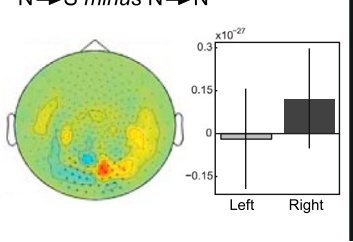

Synesthesia left, att. left $\mathrm{S} \leftarrow \mathrm{N}$ minus $\mathrm{N} \leftarrow \mathrm{N}$

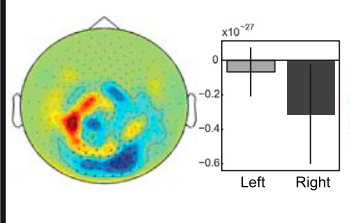

Color right, attention left $\mathrm{N} \leftarrow \mathrm{C}$ minus $\mathrm{N} \leftarrow \mathrm{N}$

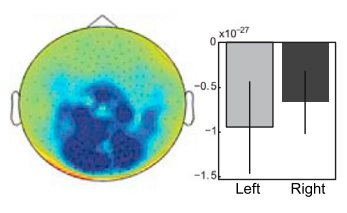

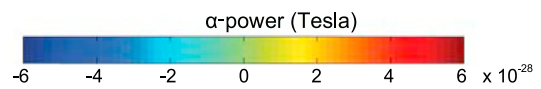

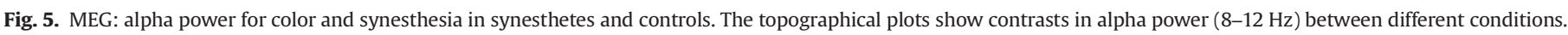

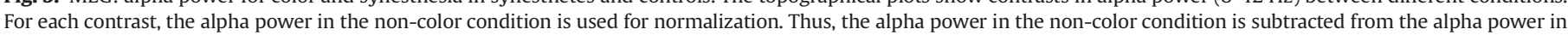

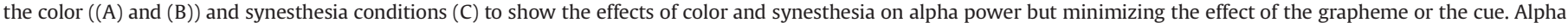

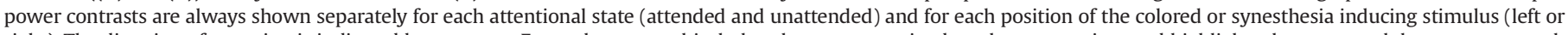

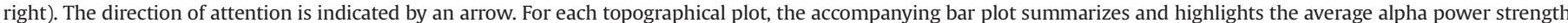

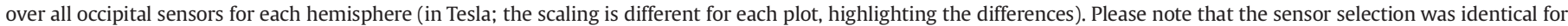

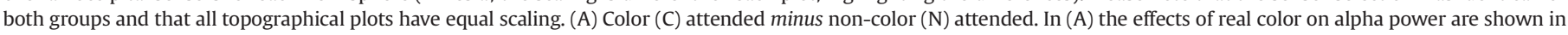

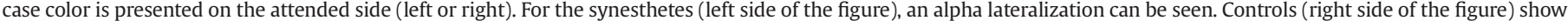

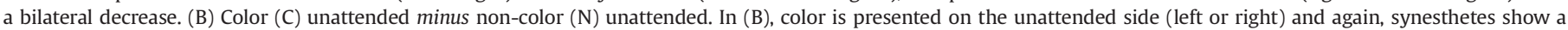

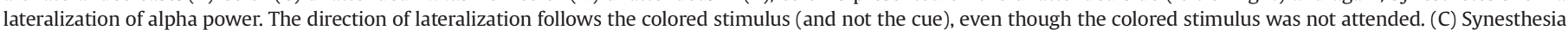

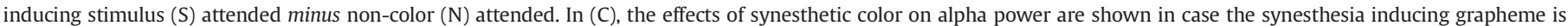

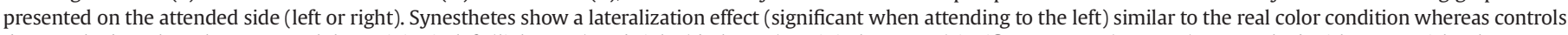

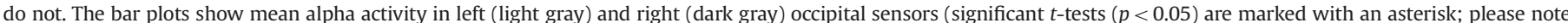

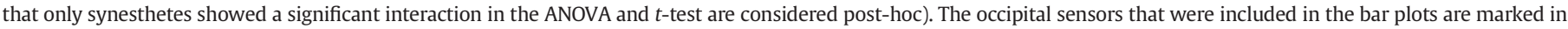
black in the topographical plots. $C=$ colored grapheme, $S=$ synesthesia inducing grapheme, $N=$ non-colored grapheme.

correlation between synesthesia strength and the lateralization in alpha power $\left(p=0.04 ; R^{2}: 0.43\right.$, slope $=-0.092 \mathrm{e}-28$; one outlier was excluded based on a threshold of 2.5 STD away from the mean). The negative correlation crossing zero shows that in synesthetes with strong effects of synesthesia alpha lateralization follows the synesthesia-inducing stimulus whereas synesthetes with weaker synesthesia show a normal alpha lateralization which follows the cue (see the schematics below Fig. 6 for details). The control group showed no correlation $\left(R^{2}=0.0015 ; p=0.9\right)$.

\section{Discussion}

This study addressed the effect of real and synesthetic colored graphemes in comparison to non-colored graphemes in synesthetes vs. controls focusing on behavioral responses as well as alpha oscillations using MEG. This paper demonstrates that both these measures (RT and alpha power) are affected differently by color percept in synesthetes vs. controls. Below we will discuss a common interpretation of our neural and behavioral findings.

The alpha inhibition theory interprets alpha power modulation as follows: alpha decreases when increased processing is required and increases for task irrelevant stimuli (e.g. Klimesch et al., 2007). Attention on the other hand can influence processing demands: processing of attended input is improved while unattended input is processed less well. The idea therefore is that (spatial) attentional effects are (partly) due to disinhibited (increased) processing at the attended site and an increased inhibition at the unattended site. Attentional perceptual effects (better or worse performance) are shown to coincide with an alpha power decrease 


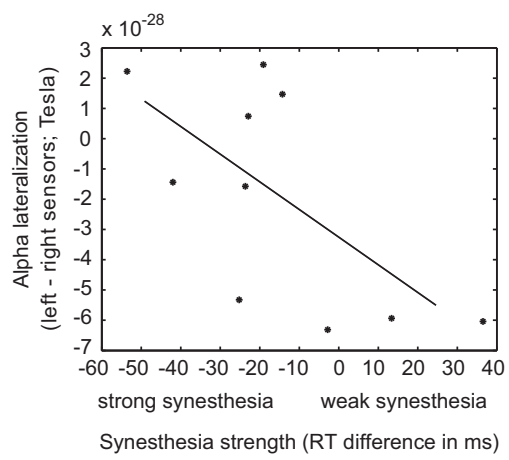

Alpha lateralization while attention is on the neutral stimulus (right - left hemifield) in the synesthesia condition:

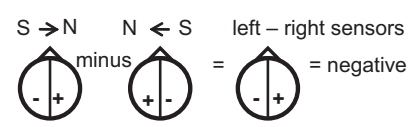

Alpha lateralization is negative if it follows the attentional cue

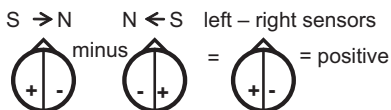

Alpha lateralization is positive if it follows the synesthetic color

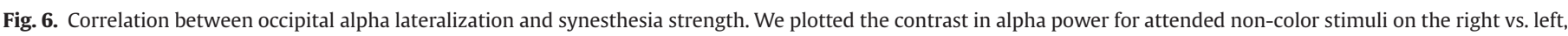

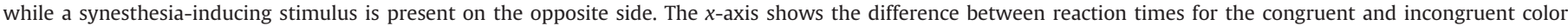

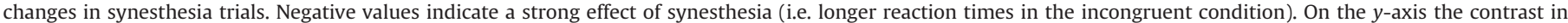

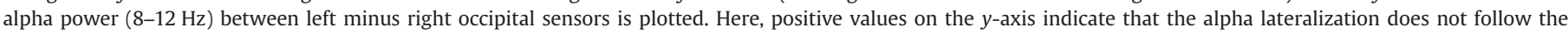

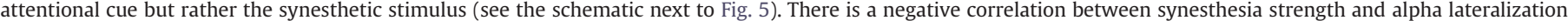
$\left(p=0.04, R^{2}: 0.43\right.$, slope $\left.=-0.092 \mathrm{e}-28\right)$.

or increase, respectively, (Kelly et al., 2006; Rihs et al., 2007; Worden et al., 2000; Yamagishi et al., 2003), suggesting a strong link between alpha oscillations and attention (e.g. Händel et al., 2011). Consistently, here we show alpha power is modulated by attention, directed by an endogenous cue, as observed in the noncolor condition. In synesthetes, the lateralization of alpha power is strengthened if color is present (contralateral alpha decrease and ipsilateral increase (Fig. 5A)). This increased lateralization implies that color strongly captures attention in synesthetes, facilitating processing at the colored location (alpha decrease) while inhibiting processing elsewhere (alpha increase). The observed alpha modulation in the uncued color condition supports this interpretation (Fig. 5B): alpha lateralization followed the color and not the attentional cue. Additionally, the presence of color decreased reaction times for the colored stimuli even though the cue was invalid.

Color is capable of automatic allocation of attention (Treisman \& Gelade, 1980) even if the predictive value is none (Busse, Katzner, \& Treue, 2006; Theeuwes \& Godijn, 2002). There is an active discussion whether color causes reaction time effects in a pure bottom-up fashion or whether these effects can be overruled by top-down influences (see e.g. Leber \& Egeth, 2006; Theeuwes, 2004). Both involuntary attentional effects of color (Busse et al., 2006; Hickey, McDonald, \& Theeuwes, 2006; Theeuwes, 2004) and top-down suppression of task irrelevant color input have been demonstrated (Bacon \& Egeth, 1994; Folk, Remington, \& Johnston, 1992; Jonides \& Yantis, 1988). Our results suggest that in synesthetes, color serves as such a strong attentional cue that it cannot be suppressed even when irrelevant for the task. Bottomup attention driven by an exogenous cue is known to act faster than top-down attention directed by endogenous cues which have to be processed and interpreted first (see e.g. Egeth \& Yantis, 1997; Müller \& Rabbitt, 1989). The arrow that was presented in our study is considered such an endogenous cue, while the presence of color induces fast feature and location specific attentional bottom-up effects (Busse et al., 2006; Theeuwes, 2004; Theeuwes \& Godijn, 2002). In synesthetes, alpha power and behavioral results indicate that these attentional effects of color are not overruled by the endogenous, task relevant attentional cue whereas in controls this is the case. However, whether color serves as bottom-up cue or, instead, induces a second top-down effect cannot be answered with our study.

In comparison, controls show a general decrease of alpha power for the color condition compared to the non-color condition. Although for controls the decrease in alpha power contralateral to the colored stimulus was stronger than the ipsilateral decrease, (Fig. 5B), there was no ipsilateral increase of alpha power. Additionally, there was no behavioral change due to color; attention always followed the cue. This qualitative difference between synesthetes and controls indicates that controls can indeed suppress the attentional capture of color using the relevant top-down cue. The spatially unspecific alpha power decrease in control participants might be explained by a general increase in alertness if color is present. Color can increase the saliency of the input (Wichmann, Sharpe, \& Gegenfurtner, 2002) and thereby affect the processing. MEG studies have shown that attention to colored input can decrease alpha activity (Müller \& Keil, 2004; Snyder \& Foxe, 2010), however, to our knowledge spatial specificity of these alpha effects has not been demonstrated.

Controls showed no effects for synesthesia-inducing stimuli while synesthetes showed similar, but weaker alpha power modulations for synesthetic color compared to real color. Albeit weaker alpha effects, the correlation between alpha lateralization and behavioral color interference due to synesthesia indicates a strong link between alpha modulation and the percept of synesthetic color. Thus, even though not as pronounced as real color, also synesthetic color attracts attention. As far as we know, the neural correlates of attentional capture by synesthesia-inducing stimuli have not been reported before.

There is an ongoing discussion whether (some) overt attention is necessary to elicit synesthesia (e.g. Edquist et al., 2006; Laeng, Svartdal, \& Oelmann, 2004; Sagiv, Heer, \& Robertson, 2006; Ward et al., 2010) or not (e.g. Carriere et al., 2009; Palmeri et al., 2002; Smilek et al., 2003). In addition to our physiological findings, our behavioral results confirm that full attention is not necessary for synesthetic percept (Mattingley, Payne, \& Rich, 2006; Rich \& Mattingley, 2003). Longer RTs in the synesthesia condition for the uncued stimulus side confirm that uncued synesthesiainducing stimuli were not fully attended. Still, for these uncued stimuli, a congruency effect of synesthetic color was observed in the reaction times. This strongly suggests that synesthesia was elicited also for not fully attended stimuli, as is supported by the correlation of alpha power and the amount of color interference.

Alpha is associated with inhibition. Can our alpha-findings be used as evidence for the disinhibition theory which predicts increased disinhibition in synesthesia (Grossenbacher \& Lovelace, 2001)? If alpha is taken as an indication of inhibition one would expect decreased alpha oscillation (i.e. increased disinhibition) in synesthetes during the synesthetic percept. A decrease in inhibition for synesthetes was demonstrated in a recent paper by Terhune, Tai, Cowey, Popescu, and Cohen Kadosh (2011), who showed evidence for increased excitability (and therefore less inhibition) in visual cortex of synesthetes in the form of lower phosphene thresholds when stimulated with transcranial 
magnetic stimulation (TMS). For synesthetes, our results however, show more pronounced alpha power effects for the real color condition compared to the synesthesia condition. Additionally, the qualitative difference between synesthetes and controls is the increased alpha power (increased inhibition) ipsilateral to the colored stimuli and not the contralateral decrease. The disinhibition hypothesis would, in contrast, predict a larger alpha decrease for synesthesia-inducing stimuli. Our alpha effects can therefore not be interpreted as directly supportive of the disinhibition theory as a mechanism specific for synesthesia. Despite our results, however, we cannot exclude the disinhibition hypothesis as a mechanism of synesthesia. We assessed oscillatory alpha power derived from occipital sensors most likely reflecting activity from multiple occipital as well as parietal regions. No specific brain regions can as such be identified. This leaves the possibility that rather subtle differences in alpha power in subregions of occipital or parietal cortex show disinhibition effects. Such effects might be difficult to identify since they can be weak, locally very confined, and additionally overlain by activity from stronger sources that do not differ between groups. Furthermore, our data does not capture the different variants of synesthesia since our subject group mainly consists of projectors (projectors $(N=10)$ and associators $(N=1)$ ). Alpha activity might show a different pattern in associators. Nevertheless, as our data are not directly supportive of the disinhibition as exclusive mechanism for synesthesia in our specific group of projector synesthetes, we propose a possible alternative mechanism of synesthesia.

Our main findings are: (i) only in synesthetes color introduces a location specific shift of attention. This is indicated by the fact that color overrides the effect of an attentional cue on alpha lateralization and RT; (ii) synesthesia-inducing stimuli lead to the same modulation of alpha power and its strength correlates with estimated synesthesia strength. These findings could simply indicate that the stronger the percept of (synesthetic or real) color, the more attention is drawn towards the (synesthetic or real) color. However, since the specific effect of color on alpha oscillations and behavior is only present in synesthetes we propose a specific relationship between color percept and synesthesia: In synesthetes, real color leads to a spatially localized, automatic change in alpha power, accompanied by an allocation of attention. If, during the development of a synesthete, a grapheme and a color are present at the same spatial location, attention will be automatically directed towards the color in a very spatially focused way, possibly facilitating binding processes between the color and the grapheme. Spatially localized attention is required for binding (e.g. form to color) (Robertson, 2003; Shafritz, Gore, \& Marois, 2002; Treisman, 2005) and spatial co-localization may enhance cross-modal coupling (Meyer, Wuerger, Rohrbein, \& Zetzsche, 2005). Once grapheme-color coupling is strengthened, processing of the grapheme could lead to coactivation of color areas. Such coupling might be easy to form at a young age (Bedny, Konkle, Pelphrey, Saxe, \& Pascual-Leone, 2010; Hensch, 2005).

Consistent with our hypothesis, synesthesia in children develops over time from non-stable grapheme-color pairings into highly consistent inducer-concurrent mappings (Hancock, 2006; Simner, Harrold, Creed, Monro, \& Foulkes, 2009); a recent paper by Witthoft and Winawer (2013) stresses the importance of learning processes for synesthesia. Once coupled, the allocation of attention also to synesthetic color could induce a positive feedback loop, strengthening functional pathways connecting the grapheme and color experience (Büchel \& Friston, 1997; Caporale \& Dan, 2008; Klink, Brascamp, Blake, \& van Wezel, 2010), eventually inducing anatomical changes in synesthetes (Bedny et al., 2010; Hensch, 2005; Noppeney, Friston, Ashburner, Frackowiak, \& Price, 2005). Anatomical differences in synesthetes have been reported (e.g. Rouw \& Scholte, 2007). However, we propose that such excess anatomical connectivity is not necessary to elicit synesthesia initially. This is consistent with the finding that synesthesia adheres to principles of normal crossmodal perception (e.g. Ward et al., 2006) and with induction of synesthesia in normal subjects (e.g. Meier \& Rothen, 2009).

Our findings are in line with recent literature that suggests altered color processing in synesthetes. Enhanced memory for color (Yaro \& Ward, 2007) and better color discrimination on a Farnsworth-Munsell color hue test were found for synesthetes (Banissy, Walsh, \& Ward, 2009). Neuroimaging studies have shown altered event-related potentials in visual EEG studies (Barnett et al., 2008; Goller, Otten, \& Ward, 2009) of which the study by Barnett and colleagues suggests that synesthetes have enhanced responsiveness in the parvocellular visual system. Structural changes in color areas in the brain have been identified as well (Banissy et al., 2012). In addition, less color-specific sensory differences have been identified (Banissy et al., 2009; Brang, Williams, \& Ramachandran, 2012). As mentioned before, Terhune et al. (2011) demonstrated that the visual cortex of synesthetes shows enhanced excitability (lower phosphene thresholds) when stimulated with transcranial magnetic stimulation (TMS). Altogether, these findings indicate that differences in early visual processing exist in synesthetes, specifically suggesting enhanced bottom-up responsiveness. This is consistent with our finding that color is special to synesthetes as it catches their attention.

Our electrophysiological and behavioral results suggest that color (real and synesthetic) automatically leads to the locationspecific allocation of attention in synesthetes, but not controls. This location-specific attention to color could explain how color is bound to the grapheme during the initial development of synesthesia. Predictions of this theory can be tested in future research. Such binding processes might also play a role outside the specific case of synesthesia. Indeed many learning and association processes might be based on automatic attention.

\section{Acknowledgements}

This work was supported by the Volkswagen-Foundation [grant number I/80743]. We would like to thank Jason Chan and Ayelet Landau for comments on the manuscript.

\section{References}

Aghajanian, G. K., \& Marek, G. J. (1999). Serotonin and hallucinogens. Neuropsychopharmacology, 21, S16-S23.

Bacon, W. F., \& Egeth, H. E. (1994). Overriding stimulus-driven attentional capture. Perception E' Psychophysics, 55, 485-496.

Banissy, M. J. Stewart, L. Muggleton, N. G. Griffiths, T. D., Walsh, V. Y. Ward, J., et al. (2012). Grapheme-color and tone-color synesthesia is associated with structural brain changes in visual regions implicated in color, form, and motion. Cognitive Neuroscience, 3, 29-35.

Banissy, M. J., Walsh, V., \& Ward, J. (2009). Enhanced sensory perception in synaesthesia. Experimental Brain Research, 196, 565-571.

Barnett, K. J., Foxe, J. J., Molholm, S., Kelly, S. P., Shalgi, S., Mitchell, K. J., et al. (2008). Differences in early sensory-perceptual processing in synesthesia: a visual evoked potential study. NeuroImage, 43, 605-613.

Baron-Cohen, S., Wyke, M. A., \& Binnie, C. (1987). Hearing words and seeing colours: an experimental investigation of a case of synaesthesia. Perception, 16 , 761-767.

Bastiaansen, M. C. M., \& Knosche, T. R. (2000). Tangential derivative mapping of axial MEG applied to event-related desynchronization research. Clinical Neurophysiology, 111, 1300-1305.

Bedny, M., Konkle, T., Pelphrey, K., Saxe, R., \& Pascual-Leone, A. (2010). Sensitive period for a multimodal response in human visual motion area MT/MST. Current Biology, 20, 1900-1906.

Berger, H. (1929). Über das Elektroenkephalogramm des Menschen. Archiv für Psychiatrie und Nervenkrankheiten, 87, 527-570.

Bien, N., ten Oever, S., Goebel, R., \& Sack, A. T. (2012). The sound of size. Crossmodal binding in pitch-size synesthesia: a combined TMS, EEG and psychophysics study. Neurolmage, 59, 663-672. 
Brang, D., Hubbard, E. M., Coulson, S., Huang, M., \& Ramachandran, V. S. (2010). Magnetoencephalography reveals early activation of V4 in grapheme-color synesthesia. Neurolmage, 53, 268-274.

Brang, D., Williams, L. E., \& Ramachandran, V. S. (2012). Grapheme-color synesthetes show enhanced crossmodal processing between auditory and visual modalities. Cortex, 48, 630-637.

Büchel, C., \& Friston, K. J. (1997). Modulation of connectivity in visual pathways by attention: cortical interactions evaluated with structural equation modelling and fMRI. Cerebral Cortex, 7, 768-778.

Busse, L., Katzner, S., \& Treue, S. (2006). Spatial and feature-based effects of exogenous cueing on visual motion processing. Vision Research, 46, 2019-2027.

Caporale, N., \& Dan, Y. (2008). Spike timing-dependent plasticity: a Hebbian learning rule. Annual Review of Neuroscience, 31, 25-46.

Carriere, J. S. A., Eaton, D., Reynolds, M. G., Dixon, M. J., \& Smilek, D. (2009). Grapheme-color synesthesia influences overt visual attention. Journal of Cognitive Neuroscience, 21, 246-258.

Cohen Kadosh, R., Henik, A., Catena, A., Walsh, V., \& Fuentes, L. J. (2009). Induced cross-modal synaesthetic experience without abnormal neuronal connections. Psychological Science, 20, 258-265.

Cohen Kadosh, R., Henik, A., \& Walsh, V. (2007). Small is bright and big is dark in synaesthesia. Current Biology, 17, R834-R835.

Corbetta, M., Shulman, G. L., Miezin, F. M., \& Petersen, S. E. (1995). Superior parietal cortex activation during spatial attention shifts and visual feature conjunction. Science, 270, 802-805.

Dixon, M. J., Smilek, D., \& Merikle, P. M. (2004). Not all synaesthetes are created equal: projector versus associator synaesthetes. Cognitive, Affective and Behavioral Neuroscience, 4, 335-343.

Edquist, J., Rich, A. N., Brinkman, C., \& Mattingley, J. B. (2006). Do synaesthetic colours act as unique features in visual search? Cortex, 42, 222-231.

Egeth, H. E., \& Yantis, S. (1997). Visual attention: control, representation, and time course. Annual Review of Psychology, 48, 269-297.

Ergenoglu, T., Demiralp, T., Bayraktaroglu, Z., Ergen, M., Beydagi, H., \& Uresin, Y. (2004). Alpha rhythm of the EEG modulates visual detection performance in humans. Cognitive Brain Research, 20, 376-383.

Esterman, M., Verstynen, T., Ivry, R. B., \& Robertson, L. C. (2006). Coming unbound: disrupting automatic integration of synesthetic color and graphemes by transcranial magnetic stimulation of the right parietal lobe. Journal of Cognitive Neuroscience, 18, 1570-1576.

Folk, C. L., Remington, R. W., \& Johnston, J. C. (1992). Involuntary covert orienting is contingent on attentional control settings. Journal of Experimental PsychologyHuman Perception and Performance, 18, 1030-1044.

Foxe, J. J., Simpson, G. V., \& Ahlfors, S. P. (1998). Parieto-occipital similar to $10 \mathrm{~Hz}$ activity reflects anticipatory state of visual attention mechanisms. Neuroreport, 9, 3929-3933.

Fu, K. M. G., Foxe, J. J., Murray, M. M., Higgins, B. A., Javitt, D. C., \& Schroeder, C. E. (2001). Attention-dependent suppression of distracter visual input can be cross-modally cued as indexed by anticipatory parieto-occipital alpha-band oscillations. Cognitive Brain Research, 12, 145-152.

Goller, A. I., Otten, L. J., \& Ward, J. (2009). Seeing sounds and hearing colors: an event-related potential study of auditory-visual synesthesia. Journal of Cognitive Neuroscience, 21, 1869-1881.

Grossenbacher, P. G., \& Lovelace, C. T. (2001). Mechanisms of synesthesia: cognitive and physiological constraints. Trends in Cognitive Sciences, 5, 36-41.

Haegens, S., Haendel, B. F., \& Jensen, O. (2011). Top-down controlled alpha band activity in somatosensory areas determines behavioral performance in a discrimination task. Journal of Neuroscience, 31, 5197-5204.

Haegens, S., Osipova, D., Oostenveld, R., \& Jensen, O. (2010). Somatosensory working memory performance in humans depends on both engagement and disengagement of regions in a distributed network. Human Brain Mapping, 31, $26-35$.

Hancock, P. (2006). Monozygotic twins' colour-number association: a case study. Cortex, 42, 147-150.

Händel, B. F., Haarmeier, T., \& Jensen, O. (2011). Alpha oscillations correlate with the successful inhibition of unattended stimuli. Journal of Cognitive Neuroscience. 23, 2494-2502.

Hanslmayr, S., Aslan, A., Staudigl, T., Klimesch, W., Herrmann, C. S., \& Bauml, K. H. (2007). Prestimulus oscillations predict between and within subjects. Neuroimage, 37, 1465-1473.

Hartman, A. M. \& Hollister, L. E. (1963). Effect of mescaline, lysergic-acid diethylamide and psilocybin on color-perception. Psychopharmacologia, 4, $441-451$.

Hensch, T. K. (2005). Critical period plasticity in local cortical circuits. Nature Reviews Neuroscience, 6, 877-888.

Hickey, C., McDonald, J. J., \& Theeuwes, J. (2006). Electrophysiological evidence of the capture of visual attention. Journal of Cognitive Neuroscience, 18, 604-613.

Hubbard, E. M., Arman, A. C., Ramachandran, V. S., \& Boynton, G. M. (2005). Individual differences among grapheme-color synesthetes: brain-behavior correlations. Neuron, 45, 975-985.

Jäncke, L., Beeli, G., Eulig, C., \& Hanggi, J. (2009). The neuroanatomy of graphemecolor synesthesia. European Journal of Neuroscience, 29, 1287-1293.

Jensen, O., Gelfand, J., Kounios, J., \& Lisman, J. E. (2002). Oscillations in the alpha band $(9-12 \mathrm{~Hz})$ increase with memory load during retention in a short-term memory task. Cerebral Cortex, 12, 877-882.

Jokisch, D., \& Jensen, O. (2007). Modulation of gamma and alpha activity during a working memory task engaging the dorsal or ventral stream. Journal of Neuroscience, 27, 3244-3251.
Jonides, J., \& Yantis, S. (1988). Uniqueness of abrupt visual onset in capturing attention. Perception \& Psychophysics, 43, 346-354.

Kelly, S. P., Lalor, E. C., Reilly, R. B., \& Foxe, J. J. (2006). Increases in alpha oscillatory power reflect an active retinotopic mechanism for distracter suppression during sustained visuospatial attention. Journal of Neurophysiology, 95, 3844-3851.

Klimesch, W., Sauseng, P., \& Hanslmayr, S. (2007). EEG alpha oscillations: the inhibition-timing hypothesis. Brain Research Reviews, 53, 63-88.

Klink, P. C., Brascamp, J. W., Blake, R., \& van Wezel, R. J. A. (2010). Experience-driven plasticity in binocular vision. Current Biology, 20, 1464-1469.

Laeng, B., Svartdal, F., \& Oelmann, H. (2004). Does color synesthesia pose a paradox for early-selection theories of attention? Psychological Science, 15, 277-281.

Leber, A. B., \& Egeth, H. E. (2006). It's under control: top-down search strategies can override attentional capture. Psychonomic Bulletin E' Review, 13, 132-138.

Mattingley, J. B., Payne, J. M., \& Rich, A. N. (2006). Attentional load attenuates synaesthetic priming effects in grapheme-colour synaesthesia. Cortex, 42, 213-221.

Meier, B., \& Rothen, N. (2009). Training grapheme-colour associations produces a synaesthetic Stroop effect, but not a conditioned synaesthetic response. Neuropsychologia, 47, 1208-1211.

Meyer, G. F., Wuerger, S. M., Rohrbein, F., \& Zetzsche, C. (2005). Low-level integration of auditory and visual motion signals requires spatial colocalisation. Experimental Brain Research, 166, 538-547.

Müller, H. J., \& Rabbitt, P. M. A. (1989). Reflexive and voluntary orienting of visualattention-time course of activation and resistance to interruption. Journal of Experimental Psychology-Human Perception and Performance, 15, 315-330.

Müller, M. M., \& Keil, A. (2004). Neuronal synchronization and selective color processing in the human brain. Journal of Cognitive Neuroscience, 16, 503-522.

Noppeney, U., Friston, K. J., Ashburner, J., Frackowiak, R., \& Price, C. J. (2005). Early visual deprivation induces structural plasticity in gray and white matter. Current Biology, 15, R488-R490.

Palmeri, T. J., Blake, R., Marois, R., Flanery, M. A., \& Whetsell, W. J. (2002). The perceptual reality of synesthetic colors. Proceedings of the National Academy of Sciences of the United States of America, 99, 4127-4131.

Ramachandran, V. S., \& Hubbard, E. M. (2001a). Psychophysical investigations into the neural basis of synaesthesia. Proceedings of the Royal Society B-Biological Sciences, 268, 979-983.

Ramachandran, V. S., \& Hubbard, E. M. (2001b). Synaesthesia-a window into perception, thought and language. Journal of Consciousness Studies, 8, 3-34.

Rich, A. N., \& Mattingley, J. B. (2003). The effects of stimulus competition and voluntary attention on colour-graphemic synaesthesia. NeuroReport, 14 1793-1798.

Rihs, T. A., Michel, C. M., \& Thut, G. (2007). Mechanisms of selective inhibition in visual spatial attention are indexed by alpha-band EEG synchronization. European Journal of Neuroscience, 25, 603-610.

Robertson, L. C. (2003). Binding, spatial attention and perceptual awareness. Nature Reviews Neuroscience, 4, 93-102.

Rothen, N., \& Meier, B. (2009). Do synesthetes have a general advantage in visual search and episodic memory? A case for group studies. PLoS One, 4, 9.

Rouw, R., \& Scholte, H. S. (2007). Increased structural connectivity in graphemecolor synesthesia. Nature Neuroscience, 10, 792-797.

Rouw, R., \& Scholte, H. S. (2010). Neural basis of individual differences in synesthetic experiences. Journal of Neuroscience, 30, 6205-6213.

Sagiv, N., Heer, J., \& Robertson, L. (2006). Does binding of synesthetic color to the evoking grapheme require attention? Cortex, 42, 232-242.

Sagiv, N., Simner, J., Collins, J., Butterworth, B., \& Ward, J. (2006). What is the relationship between synaesthesia and visuo-spatial number forms? Cognition, $101,114-128$

Shafritz, K. M., Gore, J. C., \& Marois, R. (2002). The role of the parietal cortex in visual feature binding. Proceedings of the National Academy of Sciences of the United States of America, 99, 10917-10922.

Simner, J., Harrold, J., Creed, H., Monro, L., \& Foulkes, L. (2009). Early detection of markers for synaesthesia in childhood populations. Brain, 132, 57-64.

Smilek, D., Dixon, M. J., \& Merikle, P. M. (2003). Synaesthetic photisms guide attention. Brain and Cognition, 53, 364-367.

Snyder, A. C., \& Foxe, J. J. (2010). Anticipatory attentional suppression of visual features indexed by oscillatory alpha-band power increases: a high-density electrical mapping study. Journal of Neuroscience, 30, 4024-4032.

Sperling, J. M., Prvulovic, D., Linden, D. E. J., Singer, W., \& Stirn, A. (2006). Neuronal correlates of colour-graphemic synaesthesia: a fMRI study. Cortex, 42, 295-303.

Stroop, J. R. (1935). Studies of interference in serial verbal reactions. Journal of Experimental Psychology, 18, 643-662.

Terhune, Devin B., Tai, S., Cowey, A., Popescu, T., \& Cohen Kadosh, R. (2011) Enhanced cortical excitability in grapheme-color synesthesia and its modulation. Current Biology, 21, 2006-2009.

Theeuwes, J. (2004). Top-down search strategies cannot override attentional capture. Psychonomic Bulletin \& Review, 11, 65-70.

Theeuwes, J., \& Godijn, R. (2002). Irrelevant singletons capture attention: evidence from inhibition of return. Perception \& Psychophysics, 64, 764-770.

Thut, G., Nietzel, A., Brandt, S. A., \& Pascual-Leone, A. (2006). Alpha-band electroencephalographic activity over occipital cortex indexes visuospatial attention bias and predicts visual target detection. Journal of Neuroscience, 26, 9494-9502.

Treisman, A. (2005). Synesthesia-implications for attention, binding, and consciousness. In: L. C. Robertson, \& N. Sagiv (Eds.), Synesthesia: perspectives from cognitive neuroscience (pp. 90-107). New York: Oxford University Press. 
Treisman, A. M., \& Gelade, G. (1980). Feature-integration theory of attention. Cognitive Psychology, 12, 97-136.

Van Dijk, H., Nieuwenhuis, I. L., \& Jensen, O. (2010). Left temporal alpha band activity increases during working memory retention of pitches. European Journal of Neuroscience, 31, 1701-1707.

Van Dijk, H., Schoffelen, J. M., Oostenveld, R., \& Jensen, O. (2008). Prestimulus oscillatory activity in the alpha band predicts visual discrimination ability. Journal of Neuroscience, 28, 1816-1823.

van Leeuwen, T. M., den Ouden, H. E. M., \& Hagoort, P. (2011). Effective connectivity determines the nature of subjective experience in grapheme-color synesthesia. Journal of Neuroscience, 31, 9879-9884.

van Leeuwen, T. M., Petersson, K. M., \& Hagoort, P. (2010). Synaesthetic colour in the brain: beyond colour areas. A functional magnetic resonance imaging study of synaesthetes and matched controls. PLoS One, 5, e12074.

Ward, J., Huckstep, B., \& Tsakanikos, E. (2006). Sound-colour synaesthesia: to what extent does it use cross-modal mechanisms common to us all? Cortex, 42, 264-280.

Ward, J., Jonas, C., Dienes, Z., \& Seth, A. (2010). Grapheme-colour synaesthesia improves detection of embedded shapes, but without pre-attentive 'pop-out' of synaesthetic colour. Proceedings of the Royal Society B-Biological Sciences, 277, 1021-1026.

Ward, J., Li, R., Salih, S., \& Sagiv, N. (2007). Varieties of grapheme-colour synaesthesia: a new theory of phenomenological and behavioural differences. Consciousness and Cognition, 16, 913-931.
Weiss, P. H., \& Fink, G. R. (2009). Grapheme-colour synaesthetes show increased grey matter volumes of parietal and fusiform cortex. Brain, 132, 65-70.

Weiss, P. H., Zilles, K., \& Fink, G. R. (2005). When visual perception causes feeling: enhanced cross-modal processing in grapheme-color synesthesia. NeuroImage, $28,859-868$.

Wichmann, F. A., Sharpe, L. T., \& Gegenfurtner, K. R. (2002). The contributions of color to recognition memory for natural scenes. Journal of Experimental Psychology-Learning Memory and Cognition, 28, 509-520.

Witthoft, N., \& Winawer, J. (2013). Learning, Memory, and Synesthesia. Psychological Science, 24(3), 258-265.

Wollen, K. A., \& Ruggiero, F. T. (1983). Colored-letter synesthesia. Journal of Mental Imagery, 7, 83-86.

Worden, M. S., Foxe, J. J., Wang, N., \& Simpson, G. V. (2000). Anticipatory biasing of visuospatial attention indexed by retinotopically specific alpha-band electroencephalography increases over occipital cortex. Journal of Neuroscience, 20 (RC63), 61-66.

Yamagishi, N., Callan, D. E., Goda, N., Anderson, S. J., Yoshida, Y., \& Kawato, M. (2003). Attentional modulation of oscillatory activity in human visual cortex. NeuroImage, 20, 98-113.

Yaro, C., \& Ward, J. (2007). Searching for Shereshevskii: what is superior about the memory of synaesthetes? Quarterly Journal of Experimental Psychology, 60, 681-695.

Zhang, Y., Wang, X., Bressler, S. L., Chen, Y. H., \& Ding, M. Z. (2008). Prestimulus cortical activity is correlated with speed of visuomotor processing. Journal of Cognitive Neuroscience, 20, 1915-1925. 